\title{
Arnold Flames and Resonance Surface Folds*
}

\author{
Richard P. McGehee \\ School of Mathematics \\ University of Minnesota \\ Minneapolis, MN 55455 \\ mcgehee@geom.umn.edu \\ Bruce B. Peckham \\ Department of Mathematics and Statistics \\ University of Minnesota at Duluth \\ Duluth, Minnesota 55812 \\ bpeckham@arnold.d.umn.edu
}

July 16, 2002

\begin{abstract}
Periodically forced planar oscillators are often studied by varying the two parameters of forcing amplitude and forcing frequency. For low forcing amplitudes, the study of the essential oscillator dynamics can be reduced to the study of families of circle maps. The primary features of the resulting parameter plane bifurcation diagrams are "(Arnold) resonance horns" emanating from zero forcing amplitude. Each horn is characterized by the existence of a periodic orbit with a certain period and rotation number. In this paper we investigate divisions of these horns into subregions - different subregions corresponding to maps having different numbers of periodic orbits. The existence of subregions having more than the "usual" one pair of attracting and repelling periodic orbits implies the existence of "extra folds" in the corresponding surface of periodic points in the cartesian product of the phase and parameter spaces. The existence of more than one attracting and one repelling periodic orbit is shown to be generic. For some of the families we create, the resulting parameter plane bifurcation pictures appear in shapes we call "Arnold flames." Results apply both to circle maps and forced oscillator maps.

*A similar version of this paper will appear in the International Journal of
\end{abstract} Bifurcation and Chaos. 


\section{Introduction}

Periodically forced planar oscillators arise as models of phenomena in many settings in science, engineering, and mathematics. By varying the forcing amplitude and frequency, a two-parameter family of differential equations is obtained. Such families are often studied by considering the two-parameter family of maps of the plane generated by sampling the flow of the forced oscillator differential equations at the time period of forcing. For small forcing amplitudes, the study can be further reduced to a two-parameter family of circle maps which are perturbations of rigid rotations of the circle. An exposition of this reduction is in a previous paper [McGehee \& Peckham, 1994]. We study such families in this paper; see Eq. (1) in Sec. 2.

Studies of such circle map families by Arnold [1983] and Hall [1984] indicate the presence of (Arnold) resonance horns emanating from each rational point on the zero forcing amplitude axis in the parameter plane. Each resonance horn is characterized by the existence of a periodic orbit with a certain (rational) rotation number. In the best-known example, the standard circle map family (Example 1, Sec. 2.1), at least for small enough values of the forcing amplitude, all maps corresponding to parameter values inside any one $p / q$ resonance horn have exactly two period- $q$ orbits and are all topologically conjugate. The two orbits come together in a period- $q$ saddle-node bifurcation at the boundaries of each resonance region. With the standard map, the resonance horns, along with the curves "in between" them corresponding to maps conjugate to a rigid rotation with an irrational rotation number, are the complete bifurcation story.

It is quite possible, however, to have families of circle maps whose resonance regions must be subdivided in order to respect topological equivalence. (We use resonance "region" and resonance "horn" interchangeably, often preferring resonance horn when restricting to parameter values near the tip of the full resonance region.) Such a subdivision is necessary when maps in the same period- $q$ resonance region do not all have the same number of period- $q$ orbits. The bifurcation phenomena associated with the birth and death of these extra orbits are explored in this paper.

Perhaps the most familiar parameter plane feature (but not the simplest, as we shall see) we study is the swallowtail. This feature is illustrated, for example, in two-parameter families of maps of the plane studied by Schreiber, Dolnik, Choc, and Marek [1988] and in a two-parameter family of maps of 
Figure 1: A schematic of the 3/4 resonance region from Fig. 3 of Schreiber, et. al. [1988]. The resonance region is in the $(T, A)$ parameter plane for a family of maps of the plane generated by a planar flow with "impulse forcing." The $T$ parameter can be thought of as controlling forcing frequency, the $A$ parameter as controlling forcing amplitude. Note especially the swallowtail in the $3 / 4$ resonance region.

Figure 2: (Reproduced from Fig. 6a of Frouzakis, et. al. [1991] with permission from the authors.) The $1 / 5$ resonance region in the $(k, b)$ parameter plane for a family of maps of $\mathbf{R}^{\mathbf{3}}$. Note especially the swallowtail in the resonance region.

$\mathbf{R}^{\mathbf{3}}$ studied by Frouzakis, Adomitas and Kevrekidis [1991]. Parameter plane bifurcation diagrams from those two papers are reproduced in our Figs. 1 and 2 , respectively. The Schrieber, et. al. maps are intended to be a caricature of maps of the plane generated by a periodically forced planar oscillator family; the two parameters are analogous to forcing frequency and forcing amplitude. We focus on their $3 / 4$ resonance region. It has tips at either "end" of the resonance region: one at the analogue of zero forcing amplitude $(A=0)$ and the other at a Hopf bifurcation curve (the dash-dot line in Fig. 1). The complete resonance region provides a connection between the two types of resonance horns; these two types are described in more detail in a previous paper [Peckham, 1990]. The $1 / 5$ resonance region we reproduced from the Frouzakis, et. al. paper emanates from a Hopf bifurcation curve: the straight dashed line in Fig. 2; no forced oscillator interpretation is intended. In both bifurcation diagrams, the continuation of the saddle-node curves which determine the boundary of the respective resonance regions results in swallowtail regions inside those resonance regions. The two cusps on the tail of each swallowtail are saddle-nodes with a higher order degeneracy; this cusp is different from both the forced oscillator tip and from the Hopf bifurcation tip.

Although not emphasized in either the Schrieber, et. al. paper or the Frouzakis, et. al. paper, the maps corresponding to parameter values inside the swallowtail regions have four period- $q$ orbits instead of just a single attracting-repelling pair as in the rest of each period- $q$ resonance region. Be- 
cause similar scenarios are present in the simpler setting of families of circle maps, we discuss mainly circle maps in this paper. As suggested in Aronson, McGehee, Kevrekidis, and Aris [1986], we study these scenarios by focusing on the fixed- and periodic-point surfaces in the phase $\times$ parameter space instead of just the resonance regions in the parameter space. Implications for forced oscillators are discussed briefly toward the end of the paper in Sec. 5 .

We encourage the reader to look ahead to Fig. 15 where we present our two-parameter family of circle maps whose $0 / 1$ fixed-point resonance phenomena include a swallowtail. Illustrated are the "fixed-point resonance surface" and its projection to the fixed-point "resonance horn." The curves on the fixed-point surface are along its folds (i.e., its singularities with respect to projection to the parameter plane); dynamically, these folds are saddle-node bifurcation curves. Note that when projecting to the parameter plane, the saddle-node curves either mark the boundary of the fixed-point resonance horn or the swallowtail region inside the horn. The fixed-point surface projects 2:1 outside the swallowtail region, but 4:1 inside. In other words, there is an "extra pair" of fixed points for maps corresponding to parameter values inside the swallowtail. This example will be explained further toward the end of Sec. 2, after presenting some simpler examples which serve as "building blocks" for the swallowtail example.

Most of the examples we present can be thought of as falling into one of two broad classes: those with extra pairs of orbits "at" the zero forcing amplitude horn tip, and those with extra pairs of orbits which are born only away from the horn tip. The extra orbits in the swallowtail are away from the horn tip and thus belong to the latter class. The existence of extra orbits "at" the zero forcing amplitude tip of the a resonance region may be surprising, especially to persons who are familiar with the "other" type of Arnold resonance horns: those with resonance horns originating from a Hopf bifurcation curve in a two-parameter plane of a family of maps of the plane. Arnold [1982] shows using local normal forms that in such cases (at least for periods greater than four) that maps corresponding to parameter values inside the resonance horn and near the (tip generically have only one pair of period- $q$ orbits. Thus, bifurcation features are generically possible only away from the Hopf tip of resonance regions.

In part, this paper discusses how to interpret features of a bifurcation diagram for a given family of maps. More, however, the emphasis is on generating families with desired bifurcation scenarios. In the process we 
discuss what bifurcation scenarios are possible for families of circle maps and forced oscillator maps. In Sec. 2 we provide building blocks for bifurcation scenarios and use them to generate families having bifurcation features such as the swallowtail. In Sec. 3, we extend some of our constructions from the fixed-point setting to the period- $q$ setting for $q \geq 2$. We show in Sec. 4 that the existence of subdivisions in resonance regions is generic. Implications for forced oscillators are discussed in Sec. 5.

The data for all surfaces and bifurcation curves shown in the figures in this paper were computed using the authors' continuation software [Peckham, 1988-94]. Numerical data was then viewed using the three dimensional viewing software, Geomview [Phillips et. al., 1993].

\section{Families of Circle Maps}

Consider the following family of maps of $\mathbf{R}^{\mathbf{1}}$ :

$$
x \rightarrow F_{(\phi, \alpha)}(x):=x+\phi+\alpha g_{(\phi, \alpha)}(x)
$$

where $x, \phi$, and $\alpha$ are real, and

1. $g_{(\phi, \alpha)}(x) \in C^{1}$ as a function of $\phi, \alpha$ and $\theta$, and $\left|\frac{d g_{(\phi, \alpha)}}{d x}\right| \leq 1$

2. $g_{(\phi, \alpha)}(x+2 \pi)=g_{(\phi, \alpha)}(x)$

3. $\int_{0}^{2 \pi} g_{(\phi, \alpha)}(x) d x=0$ for all $\phi$ and $\alpha$.

If we let $\theta=x(\bmod 2 \pi)$, and $f_{(\phi, \alpha)}=F_{(\phi, \alpha)}(\bmod 2 \pi)$, then $F_{(\phi, \alpha)}$ is a lift of a two-parameter family of degree one circle maps. The maps are homeomorphisms when $0 \leq \alpha \leq 1$. Such maps arise from periodically forced planar oscillators as alluded to in the introduction. Because of the relationship to periodically forced oscillators, we call the parameter $\alpha$ the forcing amplitude; the parameter $\phi$ corresponds to the forcing frequency; $g_{(\phi, \alpha)}$ is the forcing function. When $g_{(\phi, \alpha)}(x)=\sin (x)$, the resulting map of Eq. (1) is called the standard (circle map) family; see Example 1 in Sec. 2.1 below. We are primarily interested in periodic orbits of $f_{(\phi, \alpha)}$, which are usually defined in terms of the lift $F_{(\phi, \alpha)}$. 
Figure 3: Fixed points by graph. $F_{(-0.4,0.9)}(x)=x+(-0.4)+0.9\left(\left(0.7 \frac{\sin (2 x)}{2}+\right.\right.$ $0.3 \sin \left(2 \pi-h(.9) \frac{\pi}{2}\right)$, where $h(.9)=0.972$. The form of $F$ is chosen to match the form used later in the paper in Example 12.

Definition: The point $(\theta, \phi, \alpha)$ is a " $p / q$ point" (that is, $\theta$ is a $p / q$ point for $\left.f_{(\phi, \alpha)}\right)$ if $\theta:=x(\bmod 2 \pi)$ is a least-period- $q$ point for $f_{(\phi, \alpha)}$ and

$$
F_{(\phi, \alpha)}^{q}(x)=x+2 \pi p
$$

Definition: The set of $p / q$ points $\in \mathbf{S}^{1} \times \mathbf{R} \times[\mathbf{0}, \mathbf{1})$ is "the $p / q$ resonance surface." The projection of the $p / q$ resonance surface to the parameter plane is "the $p / q$ resonance horn" or "the $p / q$ resonance region."

If $\theta$ is a $p / q$ point for $f_{(\phi, \alpha)}$ with eigenvalue not equal to one $\left(\frac{\partial f^{q}}{\partial \theta}(\theta, \phi, \alpha) \neq\right.$ 1 , where $f_{(\phi, \alpha)}$ is abbreviated by $\left.f\right)$, then the implicit function theorem guarantees local continuation of the $p / q$ point in the $(\theta, \phi, \alpha)$ space as the parameters $\phi$ and $\alpha$ are varied. So we make another definition:

Definition: A $p / q$ point $(\theta, \phi, \alpha)$ with $\frac{\partial f^{q}}{\partial \theta}(\theta, \phi, \alpha)=1$ is a "singular $p / q$ point."

The most common singularities we encounter are the saddle-node bifurcation (eigenvalue 1) and the cusp bifurcation (eigenvalue 1 and a higher order degeneracy).

In this Section, we use forcing functions $g_{(\phi, \alpha)}$ which are all independent of $\phi$. Consequently, we will usually abbreviate $g_{(\phi, \alpha)}$ with $g_{\alpha}$ for the rest of Sec. 2. We will return to the more general form in Sec. 3. In this case, changing $\phi$ merely translates the circle map $f_{(\phi, \alpha)}$ and its lift $F_{(\phi, \alpha)}$. This makes the fixed-point bifurcations especially easy to understand. As $\phi$ is increased, fixed points are born at $\theta$ values corresponding to local maxima of $g_{\alpha}$; fixed points die at $\theta$ values of local minima. Usually (if $g_{\alpha}^{\prime}=0$ and $g_{\alpha}^{\prime \prime} \neq 0$ ) the births and deaths of fixed points occur in saddle-node bifurcations.

This is illustrated in Fig. 3, where we show the graphs of $y=x$ and of $F_{(\phi, \alpha)}(x)=x+\phi+\alpha g_{\alpha}(x)$ for $g_{\alpha}=0.4 \sin (x)+0.6 \frac{\sin (2 x)}{2}$, with $\phi=$ $-0.5, \alpha=0.9$. By the definition of a $0 / 1$ point, such points correspond to the intersections of the two curves. Increasing $\phi$ now translates up the graph 
of $x+\phi+\alpha g_{\alpha}(x)$. New pairs of $0 / 1$ fixed points are obviously "born" as $\phi$ increases when the values of local maxima of $\alpha g_{\alpha}(x)+\phi$ push through height zero. Similarly, pairs of orbits coalesce and "die" as values of local minima increase thru zero. In Fig. 3, one attracting-repelling pair of fixed points has already been born; as $\phi$ continues to increase, a second pair will be born, then a pair will die, and finally another pair will die, leaving no 0/1 fixed points for any larger values of $\phi$.

Restricted to $\alpha=$ constant, the $0 / 1$ resonance region occupies a line segment from $\phi=-\alpha M$ to $\phi=-\alpha m$ where $M$ and $m$ are the global maximum and global minimum, respectively, of $g_{\alpha}(x)$. This follows directly from Eq. (2). When $g_{\alpha}$ is independent of $\alpha$, the $0 / 1$ resonance region is bounded by the straight lines $\phi=-\alpha M$ and $\phi=-\alpha m$. More generally, considering $p / q$ resonance regions for $q$ 's other than one, Hall [1984] has shown that if $g_{(\phi, \alpha)}$ is independent of $\phi$ and $\alpha$ then the $p / q$ resonance horns generically open out of each point $(2 \pi p / q, 0)$ on the $\phi$ axis as "wedges," that is, the two sides of the resonance regions have different slopes. He uses the Fourier series expansion of $g(x)$ to obtain his results. The issue of how many period- $q$ orbits exist inside the period- $q$ horn is not addressed. Hall's results apply to our more general family because his results are local - the rate at which resonance horns open near the zero forcing amplitude tip of the $p / q$ resonance horn depends only on the Fourier series for the single map $g_{(p / q, 0)}(x)$.

In the following subsections, we present several example functions $g_{\alpha}$ which determine two-parameter families of circle maps via Eq. (1). The various $g_{\alpha}$ 's are chosen to illustrate the variety of features that the corresponding $0 / 1$ resonance surfaces and associated bifurcation diagrams can exhibit. We start in Sec. 2.1 with several $g_{\alpha}$ 's that are independent of $\alpha$. We focus on the number of $p / q$ orbits existing for parameter values in a $p / q$ resonance region, not just whether or not a $p / q$ orbit exists. In Secs. 2.2 through 2.6, we use the features of the Sec. 2.1 examples to construct $g_{\alpha}$ 's which do depend on $\alpha$ and whose associated bifurcation diagrams consequently are more "interesting." See also Koslovsky [1991] for two similar examples. In Sec. 3 we extend these constructions to create similar features in resonance horns for periodic orbits with periods greater than one. 
Figure 4: Example 1, the Standard Family: $g(x)=\sin (x)$. The 0/1 resonance surface and its projection to the parameter plane.

\subsection{Preliminary examples}

All the $g_{\alpha}$ 's in this subsection will be independent of $\alpha$. The resulting circle maps are defined Eq. (1). The independence of $g_{\alpha}$ on $\alpha$ implies the fixed-point (possibly degenerate) saddle-node curves are all of the form $\left\{(\theta, \phi, \alpha) \mid \theta=\theta_{c_{i}}, \phi=-V_{c_{i}} \alpha\right\}$, where $\theta_{c_{i}}$ are the critical points of $g$, and $V_{c_{i}}=g\left(\theta_{c_{i}}\right)$ are the corresponding critical values. This is because Eq. (1) implies that the curves all satisfy $F_{\left(-V_{c_{i}} \alpha, \alpha\right)}(x)=x$, and $F_{\left(-V_{c_{i}} \alpha, \alpha\right)}^{\prime}(x)=0$. The projection to the parameter plane of the two curves corresponding to the global maximum and global minimum of $g$ form the boundary of the $0 / 1$ resonance region.

Example 1: The standard family. By setting

$$
g_{\alpha}(x)=g(x)=G_{1}(x):=\sin (x)
$$

in Eq. (1), we obtain the much studied "standard family of circle maps." We restrict $\alpha$ to $0 \leq \alpha<1$ to ensure our circle maps are homeomorphisms. From Eq. (2), we see that the 0/1 resonance surface (of fixed points) is given by $\phi=-\alpha \sin x$; this projects to the $0 / 1$ resonance horn $|\phi| \leq \alpha, 0 \leq \alpha<1$. The singular $0 / 1$ points are the $0 / 1$ points with $x=\pi / 2$ or $3 \pi / 2$. These (saddle-node) curves project to $\phi=\mp \alpha$ in the parameter plane.

Figure 4 shows the $0 / 1$ fixed-point surface and its projection to the parameter plane. The forcing amplitude $\alpha$ is restricted to $[0,1] ; \theta$ is restricted to $[0,2 \pi]$; the parameter plane projection is shown at $\theta=10$. "The shading of the saddle-node curves is the same on both the surface and on the curves' parameter plane projection. The dark straight line parallel to the $\theta$ axis is a representative phase line. It is extended to the parameter plane projection to assist in identification of its parameter value. The phase portrait for this line has two fixed points at the intersection points with the $0 / 1$ surface. Successive iterates march in the positive $\theta$ direction for starting $\theta$ values "in front" of the surface ; iterates march in the negative $\theta$ direction for starting points behind the surface. The two thin straight lines parallel to the $\theta$ axis are included merely to aid in visualizing the projection to the parameter 
Figure 5: Example 1, the Standard Family: $g(x)=\sin (x)$. Several resonace surfaces and their projections to the parameter plane.

plane. This same figure format is used throughout the paper for almost all our subsequent examples.

To further place the context of our study we have included a more complete picture of resonance surfaces and the resonance regions which are their projections to the parameter space. In Fig. 5 are the $0 / 1,1 / 3,1 / 2,2 / 3$, and $1 / 1$ resonance surfaces and corresponding projections. The parameter plane bifurcation picture for this example is well known [Devaney, 1989]. It has a resonance horn corresponding to each rational number. Each resonance region, of course, has an associated resonance surface in the phase $\times$ parameter space. Since we are restricting $\alpha$ to $[0,1]$, the circle maps are all homeomorphisms and uniqueness of rotation numbers implies that none of the resonance regions may overlap. The following examples also have resonance regions corresponding to each rational number, but we are focusing only on the 0/1 resonance surfaces and regions. As we shall see in Sec. 3.1, all the features we create for a $0 / 1$ resonance can be created for any $p / q$ resonance, as well.

Example 2: One pair of extra folds - coincident projections. Let

$$
g_{\alpha}(x)=g(x)=G_{2,0}(x):=\frac{\sin (2 x)}{2} .
$$

Analogous to the standard family, the fixed point surface is given by $\phi=$ $-\alpha \frac{\sin (2 x)}{2}$; this projects to the $0 / 1$ resonance horn $|\phi| \leq \frac{\alpha}{2}, 0 \leq \alpha<1$. The singular $0 / 1$ points are the $0 / 1$ points with $x=\pi / 4,3 \pi / 4,5 \pi / 4$, or $7 \pi / 4$. The two light (saddle-node) curves correspond to the maxima of $g(x), x=\pi / 4$ and $5 \pi / 4$, and both project to $\phi=\frac{-\alpha}{2}$ in the parameter plane; The two dark

(saddle-node) curves correspond to the minima, $x=\pi / 4$ and $5 \pi / 4$, and both project to $\phi=\frac{+\alpha}{2}$ in the parameter plane. See Fig. 6. As with the standard map in Fig. 4, the dark straight line parallel to the $\theta$ axis is a representative phase line. For this example, however, we have four instead of two fixed points; two extra fixed points exist.

Example 3: One pair of extra folds - noncoincident projections. 
Figure 6: Example 2, Coincident projections: $g(x)=\sin (2 x)$.

Figure 7: Example 3, Noncoincident projections: $g(x)=0.9 \frac{\sin (2 x)}{2}+0.1 \sin (x)$.

We now construct our first example having a resonance region which must be further subdivided in order to respect topological equivalence. The subregions within the resonance region are inequivalent because they have different numbers of fixed points for the corresponding maps. In the previous example, the fact that the two maxima of $g(x)=\frac{\sin (2 x)}{2}$ are equal-valued causes the two corresponding saddle-node curves to project to the same parameter space line. This line marks the left-hand side of the fixed point resonance horn. The two equal-valued minima cause the other two saddle-node curves to project to the right-hand side of the resonance region. To separate these coincident projections, we perturb the previous example by letting

$$
g_{\alpha}(x)=g(x)=G_{2, \epsilon}(x):=(1-\epsilon) \frac{\sin (2 x)}{2}+\epsilon \sin (x) .
$$

where $0 \leq \epsilon<.5$ in order to ensure that $G_{2, \epsilon}(x)$ still has two local maxima and two local minima. The surface of $0 / 1$ points is given by $\phi=-\alpha g_{\alpha}(x)$, as in the previous two examples, but it is no longer trivial to solve analytically for the saddle-node curves in the phase $\times$ parameter space, or for their projection to the parameter plane. These bifurcation curves are, however, relatively easy to trace out numerically. The result for $\epsilon=0.1$ is Fig. 7; this should be compared to Fig. 6, which is the unperturbed $\epsilon=0$ case. The two outside saddle-node curves in the parameter plane denote the $0 / 1$ resonance horn boundaries, while the two inside curves separate the resonance horn into the center region, in which there exist two attracting and two repelling fixed points, and the two outside regions, where there exists only a single attracting-repelling pair of fixed points.

Example 4: Multiple pairs of extra folds. The previous example can easily be extended to obtain a resonance horn with $2 n$ saddle-node curves by choosing $g$ to be a periodic function with $n$ local maxima and $n$ local minima. The $n$ maxima ensure the saddle-node births of $n$ pairs of fixed points as $\phi$ increases (for $\alpha \in(0,1)$ fixed); the $n$ minima ensure $n$ saddle-node deaths. 
If the $2 n$ maximum and minimum values are distinct, the $2 n$ saddle-node curves will project to distinct lines in the parameter plane. For example, to obtain $n-1$ extra pairs of folds, we could use

$$
g_{\alpha}(x)=g(x)=G_{n, \epsilon}(x):=(1-\epsilon) \frac{\sin (n x)}{n}+\epsilon \sin (x+\tilde{B})
$$

where where $0<\epsilon<.5$ in order to ensure $G_{n, \epsilon}(x)$ has $n$ local maxima and $n$ local minima and $\tilde{B}$ is chosen to ensure all the local extremum values are distinct. This prevents curves which are distinct in the phase $\times$ parameter space from having coincident projections to the parameter space.

Example 5: An infinity of folds. By choosing $g$ to be a periodic function with an infinite number of local maxima and minima, we obtain an example with an infinite number of saddle-node curves inside the $0 / 1$ resonance region. Such an example can have an infinite number of topological equivalence classes inside the 0/1 resonance region, as well as parameter values with an infinite number of fixed points without having any interval of fixed points. We use the $C^{\infty}$ function

$$
g_{\alpha}(x)=g(x)=G_{\infty}(x):=\frac{1}{K} \sin \left(\frac{1}{x}+\tilde{B}\right) H(x / 2 \pi)
$$

where $H(x)$ is a $C^{\infty}$ function which is $C^{\infty}$ flat at $x=0$ and $x=1$ and positive on $(0,1), \tilde{B}$ is chosen so that the average value of $\sin \left(\frac{1}{x}+\tilde{B}\right) H(x / 2 \pi)$ is zero on $[0,2 \pi]$, and $K$ is the maximum derivative of $\sin (1 / x+\tilde{B}) H(x / 2 \pi)$ on $[0,2 \pi]$. Then $G_{\infty}(x)$ satisfies the three forcing function conditions listed at the beginning of Sec. 2. We choose $H(x)$ to have the form

$$
H(x)=4 h(x) h(1-x)
$$

where $h(x)$ is a $C^{\infty}$ function which is zero for $x \leq 0$ and 1 for $x \geq 1, C^{\infty}$ flat at zero and one, and strictly monotonic on $[0,1]$. (For computation throughout this paper we use the $C^{1}$ function $h(x)=3 x^{2}-2 x^{3}$, for $x \in[0,1]$.) This makes $H(x)$ symmetric about $x=.5$ where it obtains its maximum value of 1. Sketches of $h(x)$ and $H(x)$, which are also used later in the paper, are shown in Fig. 8.

No such analytic example exists since $g$ having an infinite number of local maxima or minima would force $g^{\prime}$ to be identically zero. Since $g$ is required 
Figure 8: Smooth bump functions $h(x)$ and $H(x)$.

to have average value zero (condition (3) at the beginning of Sec. 2), it must therefore be identically zero as well. This would result in a $0 / 1$ resonance horn with "width" zero: Eq. (1) indicates that if $\phi=0$ every point would be a $0 / 1$ fixed point, but if $\phi \neq 0$, no point would be a $0 / 1$ fixed point.

\section{$2.2 \quad$ Flames}

We now move to examples using functions $g_{\alpha}$ which actually depend on $\alpha$. We will use $\alpha \in[0,1]$ as a "homotopy" parameter to trade off between, for instance, the different $g$ 's in the preliminary examples of the previous section. More specifically, the examples in this subsection have the form $g_{\alpha}(x)=(1-\alpha) \tilde{g}(x)+\alpha \sin (x+\tilde{B})$ where $\tilde{g}$ has multiple maxima and minima as do Examples 2 through 5 from the previous subsection, and $\tilde{B}$ is chosen to make sure the extremum values of $g_{\alpha}(x)$ are distinct. As $\alpha$ increases from 0 to 1 , all but a single max-min pair of singularities must disappear. The disappearance generically occurs at cusps - the tips of the "flames" in the parameter space bifurcation diagrams. This is fairly immediate from Eq. (1) since the disappearance of a max-min pair for $g_{\alpha}$ implies that there is an $x$ value, say $p$, and an $\alpha$ value, say $a$, at which $g_{a}^{\prime}(p)=0$, and $g_{a}^{\prime \prime}(p)=0$; we expect that generically $g_{a}^{\prime \prime \prime}(p) \neq 0$. These conditions and Eq. (1) imply that there is a $\phi$ value, say $\phi_{0}$ such that $F_{\left(\phi_{0}, a\right)}(p)=p, F_{\left(\phi_{0}, a\right)}^{\prime}(p)=1, F_{\left(\phi_{0}, a\right)}^{\prime \prime}(p)=0$, but $F_{\left(\phi_{0}, a\right)}^{\prime \prime \prime}(p) \neq 0$, the generic conditions for a cusp bifurcation [Guckenheimer \& Holmes, 1982]. See Koslovsky [1991] for a fomal proof of the existence of the cusp.

Example 6: Single Flame. To obtain a single flame inside the 0/1 resonance horn, we define

$$
\begin{gathered}
g_{\alpha}(x):=(1-\alpha) G_{2, \epsilon}(x)+\alpha \sin (x) \\
=(1-A(\alpha)) \frac{\sin (2 x)}{2}+A(\alpha) \sin (x+B(\alpha))
\end{gathered}
$$

where $A(\alpha)=\epsilon(1-\alpha)+\alpha=\epsilon+(1-\epsilon) \alpha, B(\alpha)=0, G_{2, \epsilon}(x)$ has one extra pair of folds, as defined in Eq. (3) above, and $\epsilon \in[0,0.5)$. The second form 
Figure 9: Example 6, Single Flame: $g_{\alpha}(x)=(1-A(\alpha)) \frac{\sin (2 x)}{2}+A(\alpha) \sin (x)$, where $A(\alpha)=0.1+0.9 \alpha$. (a) The evolution of $g_{\alpha}(x): \alpha=0.1,0.5,0.9$. (b) The $0 / 1$ resonance surface and its projection to the parameter plane (with $\alpha$ replaced by $\left.h\left(\alpha^{2}\right)\right)$.

Figure 10: Example 7, Double Flame: $g_{\alpha}(x)=(1-h(\alpha)) \sin (3 x)+$ $h(\alpha) \sin \left(x+\frac{\pi}{4}\right)$. The inset shows more clearly the double flame in the projection to the parameter plane.

of $g_{\alpha}$ is given for comparison with other examples of the same form in Secs. 2.3 through 2.6; any family of that form can be viewed as a "path through the $(A, B)$ parameter space" as $\alpha$ changes. The $(A, B)$ parameter space is more fully described in Sec. 2.5 below.

Three snapshots of $g_{\alpha}(x)$ are shown in Fig. 9a for $\alpha \in[0,1]$. Note the four local extrema for $\alpha=0.1$ and only two local extrema for $\alpha=0.9$. The resulting 0/1 resonance surfaces and bifurcation diagram are shown in Fig. 9b for $\epsilon=.1$, but with $\alpha$ replaced by $h\left(\alpha^{2}\right)$, both to smooth the bifurcation pictures and to increase the size of the flame; recall the graph of $h(x)$ in Fig. 8. The $\alpha$ value of the cusp (the top tip of the flame) is the value which separates the $g_{\alpha}$ 's with two local maxima and two local minima from those with a single maximum and a single minumum.

Example 7: Multiple Flames. More generally, to have $n-1$ flames:

$$
g_{\alpha}(x):=(1-\alpha) G_{n, \epsilon}(x)+\alpha \sin (x+\tilde{B})
$$

where $\tilde{B}$ is chosen as in the definition of $G_{n, \epsilon}(x)$ in Eq. (4) above to avoid coincident projections to the phase plane fo distinct saddle-node curves.

See Fig. 10 for the 0/1 resonance surface and bifurcation diagram for a double flame created by letting $n=3, \epsilon=0$, and $\tilde{B}=\frac{\pi}{4}$; we also replaced $\alpha$ with $h(\alpha)$ for visual enhancement. The inset at the lower right shows the double flame parameter space projection more clearly.

Example 8: An infinity of Flames. Define

$$
\left.g_{\alpha}(x):=(1-\alpha) G_{\infty}(x)+\alpha \sin (x)\right)
$$


Figure 11: Example 9, Center Fold: $g_{\alpha}(x)=(1-A(\alpha)) \frac{\sin (2 x)}{2}+A(\alpha) \sin (x)$, where $A(\alpha)=1-0.7 H(\alpha)$.

where $G_{\infty}$ is the $C^{\infty}$ function with an infinite number of maxima and minima described for the infinity of folds in Example 5 above.

\subsection{Center folds}

Flames in the previous section were created by ensuring that $g_{\alpha}$ had "extra" extrema for $\alpha$ near zero, but only a single maximum and single minimum for $\alpha$ near one. If instead, as $\alpha$ increases from zero to one, we start with no extra extrema, ensure that extra max-min pairs are born by $\alpha=0.5$, and ensure that they die as $\alpha$ approaches one, we effectively "slide" the flame up out of the bottom tip of the $0 / 1$ resonance horn. Since such examples create extra folds in the $0 / 1$ resonance surface which project to the middle of the $0 / 1$ resonance horn, we call them "center folds." We create these examples simply by replacing $\alpha$ in the Basic Flames section with $1-H(\alpha)$, or any other $C^{\infty}$ function that travels from 1 to 0 to 1 as $\alpha$ increases from 0 to 1 . This has the effect of weighting the $\sin (x+B)$ term heavily for values of $\alpha$ near both zero and one, but weighting the "other" term, the one causing multiple folds, for $\alpha$ near .5. Thus, as $\alpha$ increases from zero to one, all families start with no extra folds, acquire extra folds, then lose all extra folds.

Example 9: Single Center Fold. Define

$$
\begin{aligned}
& g_{\alpha}(x):=(H(\alpha)) G_{2, \epsilon}(x)+(1-H(\alpha)) \sin (x) \\
& =(1-A(\alpha)) \frac{\sin (2 x)}{2}+A(\alpha) \sin (x+B(\alpha))
\end{aligned}
$$

where $A(\alpha)=1-(1-\epsilon) H(\alpha), B(\alpha)=0$. As with the "single flame" example, the second form of $g_{\alpha}$ is for later comparison with other examples. See Fig. 11 for the center fold with $\epsilon=0.3$.

Example 10: Multiple Center Folds For $n-1$ extra pairs of folds, define

$$
g_{\alpha}(x):=H(\alpha) G_{n, \epsilon}(x)+(1-H(\alpha)) \sin (x+\tilde{B})
$$


Figure 12: Example 12, Left-side crossing: $g_{\alpha}(x)=0.7 \frac{\sin (2 x)}{2}+.3 \sin (x+2 \pi-$ $\left.\frac{\pi}{2} h(\alpha)\right)$. (a) The evolution of $g_{\alpha}(x): \alpha=0.1,0.5,0.9$. (b) The $0 / 1$ resonance surface and its projection to the parameter plane.

where $\tilde{B}$ is chosen to have the same value as in the definition of $G_{n, \epsilon}(x)$ in Eq. (4) above.

Example 11: An infinity of Center Folds. Define

$$
g_{\alpha}(x):=(H(\alpha)) G_{\infty}(x)+(1-H(\alpha)) \sin (x)
$$

where $G_{\infty}(x)$ was defined in Example 5 above.

\subsection{Saddle-node crossings}

The emphasis on all the examples presented to this point has been controlling the births and deaths of extra pairs of fixed points. We now present some examples for which we control the crossing of saddle-node curves for families having extra pairs of orbits. The examples are constructed using the simple idea that saddle-node curves cross when values of any two local extrema for $g_{\alpha}$ cross as $\alpha$ changes.

Example 12: A single left-side crossing. Define

$$
g_{\alpha}(x):=(1-.3) \frac{\sin (2 x)}{2}+.3 \sin (x+B(\alpha))
$$

where $B(\alpha)=2 \pi-\frac{\pi}{2} h(\alpha)$. From the symmetry of the sine function, it is apparent that when $\alpha=0.5, g_{\alpha}(x)$ has two local maxima with equal values. Thus, the "crossing" takes place at $\alpha=0.5$. This is corroborated by the snapshots of $g_{\alpha}(x)$ for $\alpha=0.1,0.5,0.9$ in Fig. 12a. Note that the gobal maximum for $g_{\alpha}(x)$ switches from one local maximum to the other as $\alpha$ passes through 0.5 . Figure $12 \mathrm{~b}$ shows the $0 / 1$ resonance surface and its projection to the parameter plane.

Example 13: A "full twist" with one extra fold. Define

$$
g_{\alpha}(x):=(1-\epsilon) \frac{\sin (2 x)}{2}+\epsilon \sin (x+B(\alpha))
$$


Figure 13: Example 13, Full twist: $g_{\alpha}(x)=0.7 \frac{\sin (2 x)}{2}+.3 \sin (x+2 \pi-2 \pi h(\alpha))$.

where $B(\alpha)=2 \pi-2 \pi h(\alpha), \epsilon \in(0,0.5)$.

Since the two local maximum values are coincident at $B(\alpha)=\frac{\pi}{4}$ and $\frac{5 \pi}{4}$, and the two local minimum values are coincident at $B(\alpha)=\frac{3 \pi}{4}$ and $\frac{7 \pi}{4}$, letting $B(\alpha)$ vary from zero to $-2 \pi$, causes two left side saddle-node crossings alternating with two right side saddle-node crossings. The result for $\epsilon=0.3$ is in Fig. 13.

Example 14: A "full twist" with n-1 extra folds. This is a slight generalization of the previous example. Define

$$
g_{\alpha}(x):=(1-\epsilon) \frac{\sin (n x)}{n}+\epsilon \sin (x+B(\alpha))
$$

where $B(\alpha)=2 \pi-2 \pi h(\alpha), \epsilon \in(0,0.5)$.

\subsection{More complicated flames and folds}

We now consider bifurcation diagrams created by controlling both the saddlenode crossings and the birth/death of an extra pair of fixed points. We use the following form which has already been used for the standard map, coincident projection, noncoincident projection, single flame, single fold, left side crossing, and full twist with one extra fold examples:

$$
g_{\alpha}(x)=\frac{(1-A(\alpha))}{2} \sin (2 x)+A(\alpha) \sin (x+B(\alpha))
$$

As $A(\alpha)$ and $B(\alpha)$ vary in Eq. (5), two types of changes are effected:

1. The number of relative extrema of $g_{\alpha}$ changes from four (two maxima, two minima) to two (one maximum, one minimum) as $A(\alpha)$ increases from zero to one.

2. For $A(\alpha)$ sufficiently small to ensure $g_{\alpha}$ has four relative extrema, the values of the relative extrema cross as $B(\alpha)$ varies:

(a) The values of the two local maxima cross on the two line segments with $B=\pi / 4$ or $5 \pi / 4$; 
Figure 14: (a) The two-mode parameter space: $g_{\alpha}(x)=\frac{1-A}{2} \sin (2 x)+$ $A \sin (x)$. (b) The path through this two-mode parameter space which corresponds to the left edge swallowtail of Example 15.

Figure 15: Example 15, the left edge swallowtail: $g_{\alpha}(x)=\frac{(1-A(\alpha))}{2} \sin (2 x)+$ $A(\alpha) \sin (x+B(\alpha))$, with $A(\alpha)=1-.7 H(\alpha)$, and $B(\alpha)=2 \pi-\frac{\pi}{2} h(\alpha)$.

(b) The values of the two local minima cross on the two line segments with $B=3 \pi / 4$ or $7 \pi / 4$.

Changes in features in the bifurcation diagram for the circle maps of Eq. (1) are effected as the coefficients $(A(\alpha), B(\alpha))$ pass between the five regions of $(A, B)$ space of $[0,1] \times S^{1}$ shown in the numerically computed Fig. 14 .

When $(A(\alpha), B(\alpha))$ passes from the four-extrema region to the two-extrema region (avoiding the four cusp points of Fig. 14a), the corresponding bifurcation diagram for Eq. (1) has a cusp (a flame tip). When $(A(\alpha), B(\alpha))$ passes across an equal maximum value line (on $B=\pi / 4$ or $5 \pi / 4$ ) the bifurcation diagram for the circle map family of Eq. (1) has a crossing of saddle-node curves on the left side of its $0 / 1$ resonance region; similarly, a crossing of minima of $g_{\alpha}$ as $\alpha$ varies effects a right-side crossing of saddle-nodes.

Allowing both $A(\alpha)$ and $B(\alpha)$ to vary as $\alpha$ varies leads to bifurcation diagrams having both saddle-node crossings and cusps. The path through the $(A(\alpha), B(\alpha))$ space illustrated in Fig. 14b corresponds to Example 15 below, our left edge swallowtail example. The reader may wish to return to Examples 1, 2, 3, 6, 9, 12 and 13 to identify each one's corresponding path through the $(A, B)$ space of Fig. 14a.

Example 15: Left edge "swallowtail." This example illustrates the creation of the "swallowtail" feature mentioned in the introduction. This motivating example for our study has been constructed by combining the simple building blocks of birth/death of extra pairs of fixed points with a crossing of left side saddle-node curves. We have used Eq. (5), with $A(\alpha)=$ $1-.7 H(\alpha)$, and $B(\alpha)=2 \pi-\frac{\pi}{2} h(\alpha)$. See Fig. 15 .

Example 16: Make your own. Sketch a 0/1 resonance region bounded 
by saddle-node curves which use the following building block "moves" as $\alpha$ is increased: increasing from two to four saddle-node curves at a downward pointing cusp, decrease from four to two saddle-node curves at an upward pointing cusp, crossing two lefthand saddle node curves (only when four curves exist), and crossing two righthand saddle node curves (only when four curves exist). Parametrize a path through the AB parameter space which realizes these moves. Numerically compute the saddle-node curves to see your bifurcation creation.

\subsection{Discussion}

The form of the forcing function in Eq. (5) really includes all bifurcation diagrams attainable for any two-mode truncation of the Fourier series for $g_{\alpha}: A_{0}(\alpha)+A_{1}(\alpha) \sin \left(x+B_{1}(\alpha)\right)+A_{2}(\alpha) \sin \left(2 x+B_{2}(\alpha)\right)$. This is because $A_{0}(\alpha)$ is zero due to the zero average value condition following Eq. (1), $B_{2}(\alpha)$ can be eliminated by a translation in $\theta$, and $A_{2}(\alpha)$ can be replaced with $\frac{\left(1-A_{1}(\alpha)\right)}{2}$ because it is only the relative size of $A_{1}(\alpha)$ and $A_{2}(\alpha)$ that determines the number of relative extrema for $g_{\alpha}$. In general, bifurcations attainable from an $N$-mode truncation can be thought of as paths through a $2 N-2$ dimensional parameter space. In this sense, the standard map family is the only possibility for a one-mode truncation. Systematic generalizations to determine all possible bifurcation diagrams with truncations at higher higher modes are of course possible, but we choose only to include only a few examples with higher modes.

\section{$3 \quad$ Period- $q$ Flames}

\subsection{Equivariant examples}

All the bifurcation features described so far in the paper have been generated for the $0 / 1$ fixed-point resonance. It turns out that a slight modification allows any of our fixed-point features to appear instead for any $p / q$ resonance. Recall that all our examples have lifts of the form of Eq. (1): $F_{(\phi, \alpha)}(x):=$ $x+\phi+\alpha g_{(\phi, \alpha)}(x)$. If we replace $g_{(\phi, \alpha)}(x)$ with $\frac{g_{(\phi, \alpha)}(q x)}{q}$, we obtain a circle map that is equivariant with respect to rotations by $2 \pi p / q$. (The division by $q$ is to preserve the condition that our forcing function have derivative 
bounded by one in absolute value; recall the conditions following Eq. (1).) The lift becomes equivariant with respect to translations by $2 \pi p / q$; that is, if $\tilde{F}_{(\phi, \alpha)}(x):=x+\phi+\frac{\alpha}{q} g_{(\phi, \alpha)}(q x)$, then $\tilde{F}_{(\phi, \alpha)}\left(x+\frac{2 \pi p}{q}\right)=\tilde{F}_{(\phi, \alpha)}(x)+\frac{2 \pi p}{q}$.

From this it follows fairly easily that if $\theta$ is a fixed point for $F_{(\phi, \alpha)}$ then $\left\{\frac{x}{q}, \frac{x}{q}+\frac{2 \pi p}{q}, \ldots, \frac{x}{q}+\frac{2 \pi p(q-1)}{q}, \frac{x}{q}+2 \pi p\right\}$ is an orbit for $\tilde{F}_{\left(\frac{\phi}{q}+\frac{2 \pi p}{q}, \alpha\right)}$. That is, the orbit of $\theta=\frac{x}{q}(\bmod 2 \pi)$ under the circle map corresponding to lift $\tilde{F}_{\left(\frac{\phi}{q}+\frac{2 \pi p}{q}, \alpha\right)}$ is a $p / q$ orbit. This correspondence between $0 / 1$ points of the circle map with lift $F_{(\phi, \alpha)}$ and $p / q$ orbits with lift $\tilde{F}_{\left(\frac{\phi}{q}+\frac{2 \pi p}{q}, \alpha\right)}$ turns out to be one-to-one whenever the equivariant lift $F_{(\phi, \alpha)}$ is a homeomorphism; we can ensure $F_{(\phi, \alpha)}$ is a homeomorphism by restricting $\alpha$ to be in $[0,1]$.

Consequently, all the least-period- $q$ resonance regions for $\tilde{F}$ will have exactly the same bifurcation features as the $0 / 1$ resonance region for $F$. The only difference is that the $p / q$ resonance regions for $\tilde{F}$ will be contracted by a factor of $\frac{1}{q}$ in the horizontal $(\phi)$ direction. Thus any bifurcation feature from Sec. 2 can be made to appear in any $p / q$ resonance region.

\subsection{General examples}

Analytic studies of period- $q$ bifurcation phenomena for $q>1$ are much more difficult for general lifts of circle maps of the form $F_{(\phi, \alpha)}(x):=x+\phi+$ $\alpha g_{(\phi, \alpha)}(x)$ where $g_{(\phi, \alpha)}(x)$ lacks the special structure of the above subsection which led to $F$ (called $\tilde{F}$ in the above subsection) being equivariant. We can, however, show that when the forcing amplitude $\alpha$ is small, only the Fourier modes of $g_{(\phi, \alpha)}$ which are multiples of $q$ affect whether or not "extra" folds exist on a $p / q$ resonance surface as it emanates from the circle $\left(\frac{p}{q}, 0, \theta\right)$ for $\theta \in[0,2 \pi)=S^{1}$ in the three-dimensional phase $\times$ parameter space. Unfortunately, the analysis is to lowest order terms in $\alpha$, so it guarantees nothing about the number of extra folds for values of $\alpha$ away from zero.

The low forcing amplitude analysis is still of use to us in at least the following two contexts. First, we can combine it with some results from complex dynamics to create examples with the guarantee that any extra folds that exist on any $p / q$ resonance surface will disappear as $\alpha$ increases sufficiently close to $\alpha=1$; this will in turn guarantee the existence of flames in the corresponding $p / q$ resonance regions. We present this construction following the low forcing amplitude analysis. Second, the low forcing amplitude analysis 
helps us to show that the existence of extra folds on resonance surfaces is a generic property of circle maps whose lifts are given by Eq. (1) and therefore a generic property for periodically forced oscillator families. This program is detailed in Sec. 4.

\subsubsection{Low forcing amplitude analysis}

We assume the starting point is the lifted circle map of Eq. (1). We use subscripts on the $x$ variable to indicate iterations:

$$
x_{n+1}=F_{(\phi, \alpha)}\left(x_{n}\right)=x_{n}+\phi+\alpha g_{(\phi, \alpha)}\left(x_{n}\right)
$$

Straightforward computation leads to:

$$
x_{n}=x_{0}+n \phi+\alpha \sum_{k=0}^{n-1} g_{(\phi, \alpha)}\left(x_{k}\right)
$$

Since the existence of a $p / q$ point requires $x_{q}=x_{0}+2 \pi p$, as in Eq. (2), we require

$$
q \phi+\alpha \sum_{k=0}^{q-1} g_{(\phi, \alpha)}\left(x_{k}\right)=2 \pi p
$$

To obtain a power series expansion around the parameter point $(\phi, \alpha)=$ $\left(\frac{2 \pi p}{q}, 0\right)$, we replace the parameter $\phi$ with the small parameter $\epsilon$ via $\phi=$ $\frac{2 \pi p}{q}+\epsilon$.

So Eq. (6) becomes

$$
q \epsilon+\alpha \sum_{k=0}^{q-1} g_{\left(\frac{2 \pi p}{q}+\epsilon, \alpha\right)}\left(x_{k}\right)=0
$$

Using the Fourier expansion $g_{\left(\frac{2 \pi p}{q}+\epsilon, \alpha\right)}(x)=\sum_{n=-\infty}^{\infty} c_{n}(\epsilon, \alpha) e^{i n x}$, switching the order of summation, noting that $\sum_{k=0}^{q-1} e^{\frac{i n k 2 \pi p}{q}}$ is $q$ for $n=m q$ and 0 otherwise, alters Eq. (7) to

$$
q \epsilon+q \alpha \sum_{m=-\infty}^{\infty} c_{m q}(0,0) e^{i q m x_{0}}+O\left(\alpha^{2}, \alpha \epsilon\right)=0
$$

By the implicit function theorem, Eq. (8) can be solved for $\epsilon$ as a function of $\alpha$ and $x_{0}$ : 


$$
\epsilon=-\alpha \sum_{m=-\infty}^{\infty} c_{m q}(0,0) e^{i q m x_{0}}+O\left(\alpha^{2}\right)
$$

Hall [1984] used Eq. (9) to conclude that period- $q$ resonance horns generically open as wedges, not cusps. That is, the sides of the resonance horns are not tangent at the $(\phi, \alpha)=(p / q, 0)$ tip. This follows from the generic assumption that not all of the coefficients of form $c_{ \pm n q}$ are zero. As pointed out to us by Hall, the standard map, and all the examples of this paper for which $g_{(p / q, 0)}(x)$ has only a finite number of terms in its Fourier expansion, are nongeneric in this sense: all coefficients of form $c_{ \pm n q}$ are zero for all $q$ 's larger than the largest mode with nonzero coefficient in its Fourier expansion. For the standard map, this nongenericity is manifested in every non-fixedpoint resonance horn: they all open as cusps, not wedges. This is consistent with Fig. 5.

\subsubsection{Flames in $p / q$ resonance horns}

We use Eq. (9) to interpret how many period- $q$ orbits there are for various parameter values. More specifically, if we fix $\alpha$ and vary $x_{0}$ from zero to $2 \pi$, the projection to the $(\epsilon, \alpha)$ parameter space travels back and forth in the $\epsilon$ direction along a line segment. The endpoints of the line segment are on the boundary of the $p / q$ resonance horn in the parameter space. The number of period- $q$ points for any parameter value on this line segment is equal to the number of times we pass through that parameter point as $x_{0}$ varies from zero to $2 \pi$. The number of period- $q$ orbits is the number of period- $q$ points divided by $q$. Except at $\epsilon$ parameter values ( $\alpha$ is still fixed) which correspond to critical values for $\epsilon$ as a function of $x_{0}$ via Eq. (9), such as at saddle-node orbits, the number of period- $q$ orbits will be divisible by 2 . One attracting-repelling pair of orbits is the minimum possible number. Any further pairs correspond to extra pairs of periodic orbits. (Note: There isn't a natural fixed pairing of attracting with repelling periodic orbits except for orbits near a saddle-node bifurcation; the term pair is therefore being loosely used.)

With this in mind, we note that, for $\alpha$ and $\epsilon$ small enough, there will be $n-1$ extra pairs of $p / q$ orbits if $g_{(\phi, \alpha)}$ has a Fourier series at $(\phi, \alpha)=(p / q, 0)$ for which coefficients $c_{ \pm n q}$ sufficiently dominate the coefficients $c_{ \pm m q}, m \neq n$. If $g_{(\phi, \alpha)}$ is independent of $\phi$, then the small $\alpha$ part of all $p / q$ resonance regions 
may be studied at once since the Fourier series for $g_{(p / q, 0)}$ is the same for all $p / q$. For example, if $g_{(\phi, \alpha)}(x)=\sin (6 x)$, the fixed point surface will have 5 extra fixed point pairs (6 total pairs) resulting in 10 extra folds (12 total folds) on the $p / 1$ surfaces; the period-two surface will have 2 extra period- 2 orbits resulting in 8 extra folds on the $p / 2$ surfaces; the period-three surface will have one extra pair of orbits resulting in 6 extra folds on the $p / 3$ surfaces; and the period- 6 surfaces will have no extra fold pairs.

The consequence of the above discussion is that we can control the number of extra orbits near the $(p / q, 0)$ tip of the $p / q$ resonance horn. Unfortunately, since the period- $q$ analysis was "local," it says nothing about controlling the number of pairs of orbits which exist for higher values of $\alpha$. We can, however, obtain some control by utilizing some results from complex dynamics. The following lemma was communicated to us by R. L. Devaney, N. Fagella and G. R. Hall; the first published version we are aware of (with essentially the same proof) appears in Yakobson [1985].

Lemma : Let $f_{(\phi, \alpha)}$ be the standard family: $f_{(\phi, \alpha)}(\theta)=\theta+\phi+\alpha \sin (\theta)$. Then for any fixed values of $\phi$ and $\alpha$ with $\alpha \in[0,1)$, the corresponding circle map $f_{(\phi, \alpha)}$ can have at most one attracting periodic orbit.

Sketch of proof: Consider the complex family $S_{(\phi, \alpha)}(z)=z e^{i \alpha} e^{\frac{\beta}{2}\left(z-\frac{1}{z}\right)}$. The family $S_{(\phi, \alpha)}$ restricted to the unit circle is the real standard map $f_{(\phi, \alpha)}$ [Fagella, 1994]. Each $S_{(\phi, \alpha)}$ is also a complex analytic map on $\mathbf{C} \backslash\{\mathbf{0}\}$ and therefore any attracting orbit must attract a critical point [Devaney, 1989]. Each $S_{(\phi, \alpha)}$ has critical points satisfying $\alpha z^{2}+2 z+\alpha^{2}=0$. For $\alpha<1$, these critical points, $c_{1}$ and $c_{2}$, are on the negative real axis and satisfy $c_{2}=\frac{1}{\overline{c_{1}}}$. Since $S(1 / \bar{z})=1 / \overline{S(z)}$, the two critical orbits are "symmetric with respect to the unit circle." Thus, any attracting periodic orbit must attract not just one, but both critical points. So there can exist only one attracting periodic orbit.

This enables us to construct a single example with flames in both a $p_{1} / q_{1}$ and a $p_{2} / q_{2}$ resonance horn.

Example 19: Flames for two resonances. Define 


$$
g_{\alpha}(x)=(1-\alpha)\left(\frac{\sin \left(n_{1} q_{1} x\right)}{q_{1}}+\frac{\sin \left(n_{2} q_{2} x\right)}{q_{2}}\right)+\alpha \sin (x)
$$

If $q_{1}$ and $q_{2}$ are relatively prime, this example will have $n_{1}-1$ extra pairs of $p_{1} / q_{1}$ orbits, causing $2\left(n_{1}-1\right) q$ extra folds in the $p_{1} / q_{1}$ resonance surface for small $\alpha$; similarly, there will be $n_{2}-1$ extra pairs of $p_{2} / q_{2}$ orbits for small $\alpha$. Because any extra orbits must disappear as $\alpha$ increases toward one (see the above Lemma), both the $p_{1} / q_{1}$ and $p_{2} / q_{2}$ resonance regions will have flames.

Example 19 could be easily generalized to create flames in any finite number of resonance regions whose periods are all relatively prime. The extreme case is a the following single example having flames in an infinity of resonance regions. The price, however, is that only a finite number of flames will necessarily persist under perturbation.

Example 20: Flames in an infinity of horns. We now present an analytic example having an infinite number of resonance horns with flames. Define

$$
g_{\alpha}(x)=(1-\alpha) g_{0}(x)+\alpha \sin (x)
$$

where $g_{0}(x)$ is a $C^{\omega}$ map causing the associated family of circle maps, $F_{(\phi, \alpha)}=$ $x+\phi+\alpha g_{0}(x)$ to have infinitely many horns with extra folds. Thus, as $\alpha$ increases from zero to one, the circle maps corresponding to the form of $g_{\alpha}$ for Example 20 will have "extra" folds in the infinity of horns which must all die out, forming the infinitely many flame tips.

To define $g_{0}(x)$, we first let $r$ be any real number. We will construct $g_{0}$ so that the corresponding family of circle maps has a $p / q$ horn with a flame for an infinite sequence of $p / q$ 's which accumulate on $r$. First choose a sequence $\left\{\frac{p_{n}}{q_{n}}\right\} \rightarrow r$. Require also that the $q_{n}$ 's are all relatively prime. Then define $g_{0}$ by prescribing its Fourier series coeffients to be $c_{ \pm 2 q_{n}}=e^{-2 q_{n}}$ for all $q_{n}$ 's in the chosen sequence of $\frac{p_{n}}{q_{n}}$ 's, and zero for all other coefficients. Such a function is $C^{\omega}$ since the Fourier series coefficents decay exponentially [Wheeden \& Zygmund, 1977]. (This follows from the fact that, after complexifying $g_{0}(x)$ to $g_{0}(z)$, we have that $g_{0}(z)=F \circ G(z)$, where $G(z)=e^{i z}$ is entire and $F(w)=$ $\sum_{k=-\infty}^{\infty} c_{k} w^{k}$ converges, by our choice of $c_{k}$ 's, for $|w|<e$. In particular, $g_{0}(z)$ is analytic for $z$ real, since $\left|e^{i x}\right|=1<e$.) As in the previous example, each 
period- $q_{n}$ surface for $x_{n+1}=f\left(x_{n}\right)=x_{n}+\phi+\alpha g_{\alpha}(x)$ has an "extra" pair of period $-q_{n}$ orbits associated with it if $0<\alpha<<1$, but no extra orbits for $0<<\alpha<1$. Consequently, every $\frac{p_{n}}{q_{n}}$ horn will have a flame in it.

\section{$4 \quad$ Flames are Generic}

Let $\Upsilon$ be the function space of lifts of two-parameter families of circle homeomorphisms of the form of Eq. (1): $F_{(\phi, \alpha)}(x)=x+\phi+\alpha g_{(\phi, \alpha)}(x)$. Assume the dependence of the homeomorphisms is $C^{\infty}$ as a function defined on the cartesian product of the phase space $\mathbf{R}$ with the $(\phi, \alpha)$ parameter plane. Endow $\Upsilon$ with the $C^{\infty}$ topology [Ruelle, 1989]. We now show that the property of having only one attracting and one repelling orbit in each $p / q$ resonance region (as with the standard family) is nongeneric for two-parameter families in $\Upsilon$. Equivalently, the property of having resonance regions with internal bifurcation features is shown to be generic. The rest of this section is devoted to this task.

Theorem: There is an open dense set of families in $\Upsilon$ having the property that the family has at least two hyperbolic attracting and two hyperbolic repelling period- $q$ orbits in at least part of one $p / q$ resonance region.

We will need the following two lemmas for the proof of the theorem.

Lemma 1: Let $F_{(\phi, \alpha)}(x) \in \Upsilon$. Let $p / q$ be any reduced rational number. Since $g$ is at least $C^{1}, g_{(p / q, 0)}(x)$ equals its Fourier series expansion [Wheeden \& Zygmund, 1977] which we write in the following form (the average value zero condition on $g$ forces the constant term to be zero):

$$
g_{(p / q, 0)}(x)=\sum_{k=1}^{\infty} A_{k} \sin \left(k x+B_{k}\right)
$$

If $2 q\left|A_{2 q}\right|>q\left|A_{q}\right|+\sum_{k=3}^{\infty} k q\left|A_{k q}\right|$ then for $\alpha$ a fixed positive but sufficiently small number, the $p / q$ resonance region for $F_{(\phi, \alpha)}$ will include an open interval of $\phi$ values for which the corresponding circle map has at least four $p / q$ orbits.

Proof: Define $\tilde{g}(x)=\sum_{k=1}^{\infty} A_{k q} \sin \left(k q x+B_{k}\right)$. Recall that $\epsilon=0$ in Eq. (9) corresponds to $\phi=p / q$. That equation also shows that only the Fourier 
modes of $g_{(p / q, 0)}(x)$ which are multiples of $q$ need to be considered to determine the existence of extra orbits near $\alpha=0$. This is why we defined $\tilde{g}(x)$ as we did.

Note that $\frac{d}{d x}\left(A_{2 q} \sin \left(2 q x+B_{2 q}\right)\right)=2 q A_{2 q} \cos \left(2 q x+B_{2 q}\right)$ which equals $2 q A_{2 q}$ for $x=\frac{2 \pi k-B_{2 q}}{2 q}, k=0, \ldots, 2 q-1$, and equals $-2 q A_{2 q}$ for $x=\frac{2 \pi k+\pi-B_{2 q}}{2 q}, k=$ $0, \ldots, 2 q-1$. The inequality in the hypotheses of the lemma guarantees that the magnitude of the contribution to $\tilde{g}^{\prime}(x)$ due to the sum of the rest of the Fourier modes in $\tilde{g}(x)$ is dominated at the $4 q$ points just listed by the contribution to $\tilde{g}^{\prime}(x)$ due to the $2 q^{\text {th }}$ mode. Thus, for $x \in[0,2 \pi), \tilde{g}(x)$ must have at least $2 q$ points with positive slopes alternating with $2 q$ points having negative slopes.

To show that this implies the existence of at least $4 q$ distinct $p / q$ orbits for positive values of $\alpha$, note first that Eq. (9) can be rewritten (dropping the subscript on $x_{0}$ ) as

$$
\epsilon_{\alpha}(x)=-\alpha \tilde{g}(x)+O\left(\alpha^{2}\right) .
$$

By fixing $\alpha$ sufficiently small, Eq. (11) implies that $\epsilon_{\alpha}$ has $2 q$ points with positive slopes alternating with $2 q$ points having negative slopes for $x \in$ $[0,2 \pi)$.

Since all points on a period- $q$ orbit must exist at the same parameter value, we must have $\epsilon(x)=\epsilon\left(\left(F_{(\epsilon(x), \alpha)}(x)\right)\right.$. Thus it is sufficient to consider the graph of $\epsilon_{\alpha}(x)$ only on a "fundamental interval," say for $x \in\left[0, F_{(\epsilon(0), \alpha)}(0)\right)$. On this fundamental interval there must exist two points with positive slopes alternating with two points with negative slopes. Straightforward calculus arguments lead to the existence of two local maxima and two local minima on the fundamental interval, with the two local maxima both strictly larger that both local mimima. That is, there exist $\epsilon$ values $M_{1} \geq M_{2}>m_{2} \geq m_{1}$, where the $M_{i}$ 's are local maxima and the $m_{i}$ 's are local mimima. For any $\epsilon_{0} \in\left(m_{2}, M_{2}\right)$, this forces at least four $x$ values in the fundamental interval $\left[0, F_{(\epsilon(0), \alpha)}(0)\right)$ to satisfy $\epsilon(x)=\epsilon_{0}$. These four $x$ values represent four distinct $p / q$ orbits, so $\left(m_{2}, M_{2}\right)$ is the open interval of $\phi$ values claimed to be in the $p / q$ resonance region for our fixed small value of $\alpha$.

Lemma 2: Let $g(x)=\sum_{k=1}^{\infty} A_{k} \sin \left(k x+B_{k}\right)$ be a $C^{\infty}$ function on $\mathbf{S}^{1}$. Let $N$ be an open neighborhood of $g$ in the $C^{\infty}$ topology. Then there exists 
a $\tilde{g} \in N$ and a $q \in \mathbf{Z}$ such that if $\tilde{g}(x)=\sum_{k=1}^{\infty} \tilde{A}_{k} \sin \left(k x+\tilde{B}_{k}\right)$, then $2 q\left|\tilde{A}_{2 q}\right|>q\left|\tilde{A}_{q}\right|+\sum_{k=3}^{\infty} k q\left|\tilde{A}_{k q}\right|$.

Proof: By the definition of the $C^{\infty}$ topology ([Ruelle, 1989, Appendix B], for example), any $C^{\infty}$ neighborhood contains a $C^{r}$ neighborhood for some positive integer $r$. That is, there exists a $r_{1} \in \mathbf{Z}^{+}$and an $\epsilon_{1}>0$ such that $\left\{\tilde{g}:\|\tilde{g}-g\|_{r_{1}}<\epsilon_{1}\right\} \subset N$, where $\|\tilde{g}-g\|_{r_{1}}=\max _{0 \leq r \leq r_{1}}\left\|\tilde{g}^{(r)}-g^{(r)}\right\|$ and $\left\|\tilde{g}^{(r)}-g^{(r)}\right\|=\max _{x \in[0,2 \pi]}\left|\tilde{g}^{(r)}(x)-g^{(r)}(x)\right|$. Recall also that $g \in C^{r}$ implies $\left|A_{k}\right|=o\left(\frac{1}{k^{r}}\right)$ [Zygmund, 1968] and $g \in C^{\infty}$ implies $g \in C^{r}$ for all nonnegative integers $r$. Therefore, $g \in C^{r_{1}}$ and so

$$
\left|A_{k}\right| k^{r_{1}} \rightarrow 0 \text { as } k \rightarrow \infty
$$

Now choose $q \in \mathbf{Z}^{+}$large enough so that $\left|A_{k}\right| k^{r_{1}}<\frac{1}{2} \frac{\epsilon_{1}}{2^{r_{1}}}$ for all $k>q$. Let $A=\frac{1}{2} \frac{\epsilon_{1}}{2^{r_{1}} q^{r_{1}}}$. This choice of $A$ implies that for all for all $k>q$ :

$$
\frac{\left|A_{k}\right| k^{r_{1}}}{q^{r_{1}}}<A<\frac{\epsilon_{1}}{2^{r_{1}} q^{r_{1}}}
$$

Define $\tilde{A}=\operatorname{sgn}\left(A_{2 q}\right) A$. $\quad\left(A_{2 q}\right.$ is defined from the Fourier series for $g$ in the statement of Lemma 2.) Define $\tilde{B}=B_{2 q}$. Finally define $\tilde{g}(x)=$ $g(x)+\tilde{A} \sin (2 q x+\tilde{B})$.

To show $\tilde{g} \in N:\|\tilde{g}-g\|_{r_{1}}=\|\tilde{A} \sin (2 q x+\tilde{B})\|_{r_{1}}=(2 q)^{r_{1}} A<\epsilon_{1}$ by the second inequality in Eq. (13). (In the case of the analytic topology instead of the $C^{\infty}$ topology, the $C^{0}$ norm can be used, but $x$ must be allowed to be complex. Then $\|\tilde{g}-g\|_{0}=\|\tilde{A} \sin (2 q x+\tilde{B})\|_{0}<2 A<\epsilon_{1}$ since $|\sin (2 q z+\tilde{B})|<2$ for $z$ in a sufficiently small neighborhood of the real line. See the notes at the end of this Section.)

To show the required dominance of the $\tilde{A}_{2 q}$ term over the rest of the $\tilde{A}_{k q}$ terms:

$$
\begin{gathered}
q\left|\tilde{A}_{q}\right|+\sum_{k=3}^{\infty} k q\left|\tilde{A}_{k q}\right|=q\left|A_{q}\right|+\sum_{k=3}^{\infty} k q\left|A_{k q}\right| \leq \sum_{k=1}^{\infty} k q\left|A_{k q}\right| \\
<\sum_{k=1}^{\infty} k q \frac{A q^{r_{1}}}{(k q)^{r_{1}}} \text { by the first inequality in Eq. (13) } \\
=A q \sum_{k=1}^{\infty} \frac{1}{k^{r_{1}-1}}<2 A q \\
\leq 2 q\left|\tilde{A}_{2 q}\right|, \text { since }\left|\tilde{A}_{2 q}\right|=A+|\tilde{A}|
\end{gathered}
$$


Now we are in a position to prove the genericity of flames:

Proof of the theorem: The openness in $\Upsilon$ of the families with a resonance region having at least two hyperbolic attracting and two hyperbolic repelling orbits follows from the structural stability of hyperbolic orbits. So we need only verify the denseness of such families in $\Upsilon$. Since families of the form of Eq. (1) differ only via their respective forcing functions, $g_{(\phi, \alpha)}$, the function space $\Upsilon$, defined at the beginning of this section, is equivalent to the function space $\Upsilon_{1}$, defined as the function space of smooth two-parameter families of smooth forcing functions. Thus, for a given forcing function, $g_{(\phi, \alpha)}$, and a neighborhood $N_{1}$ of $g_{(\phi, \alpha)}$ in $\Upsilon_{1}$, we must produce a $\tilde{g}_{(\phi, \alpha)}$ in $N_{1}$ whose corresponding family of circle homeomorphisms will have a $p / q$ resonance region having at least two attracting and two repelling period- $q$ orbits.

The strategy is to choose some resonance $p_{1} / q_{1}$ and determine a perturbation as in Lemma 2, with $g_{\left(p_{1} / q_{1}, 0\right)}(x)$ playing the role of $g$ in Lemma 2, which guarantees via Lemma 1 that the $p_{1} / q_{1}$ resonance region has the desired extra orbits. This perturbation can either be applied to $g_{(\phi, \alpha)}$ for all values of $\phi$ and $\alpha$, or just to maps having parameter values in a neighborhood of $\left(p_{1} / q_{1}, 0\right)$. But there is a technical glitch: $q_{1}$ must be greater than the value of $q$ that is chosen by Lemma 2 , but $q$ is determined only after the Fourier series for $g_{\left(p_{1} / q_{1}, 0\right)}$ is known. So we need a uniform choice of $q$ that will enable us to proceed with Lemma 2 for any forcing function $g_{(\phi, 0)}$ with $\phi$ in a nontrivial interval. Then we can choose a rational number $p_{1} / q_{1}$ in this $\phi$ interval with $q_{1}>q$ and perturb to obtain the desired extra $p_{1} / q_{1}$ orbits. An additional arbitrarily small perturbation can be made (by Sard's Theorem), if necessary, to ensure two orbits are hyperbolically attracting and two are hyperbolically repelling. So the following lemma completes the proof.

Lemma 3: Let $g_{\mu}(x), \mu \in \mathbf{R}$ be a $C^{0}$ family of $C^{r+1}, r \in \mathbf{Z}^{+}$, periodic functions having Fourier series expansions:

$$
g_{\mu}(x)=\sum_{k=1}^{\infty} A_{k}(\mu) \sin \left(k x+B_{k}(\mu)\right) .
$$

Let $K$ be a compact set in $\mathbf{R}$. Then $\left|A_{k}(\mu)\right| k^{r} \rightarrow 0$ as $k \rightarrow \infty$ uniformly for 
$\mu \in K$.

Proof: Note that convergence for each fixed $\mu$ is already established for $C^{r}$ periodic functions by Eq. (12). From Wheeden \& Zygmund [1977], we have that $f \in C^{1}$ implies

$$
\left|A_{k}\right| \leq \frac{1}{2 \pi} \int_{0}^{2 \pi}\left|f(x)-f\left(x+\frac{\pi}{k}\right)\right| d x \leq\left\{\max _{x \in \mathbf{S}} f^{\prime}(x)\right\} \frac{\pi}{k} .
$$

By applying this inequality to $g_{\mu}^{(r)}(x)$ whose Fourier series expansion is

$$
\sum_{k=1}^{\infty} k^{r} A_{k}(\mu) \sin \left(k x+B_{k}(\mu)\right)
$$

we obtain

$$
\left|A_{k}(\mu)\right| k^{r} \leq\left\{\max _{x \in \mathbf{S}, \mu \in \mathbf{K}} g_{\mu}^{(r+1)}(x)\right\} \frac{\pi}{k} .
$$

Since $g_{\mu} \in C^{r+1}$, the maximum in this expression exists and is finite, so the uniform convergence of $\left|A_{k}(\mu)\right| k^{r}$ to zero is established.

Notes about replacing the $C^{\infty}$ topology in the Theorem:

1. Parameter dependence need not be $C^{\infty}$, only $C^{2}$. $C^{2}$ dependence is necessary to use the implicit function theorem in going from Eq. (8) to Eq. (9).

2. The theorem's result is also true if the family of homeomorphisms is assumed to be analytic and $\Upsilon$ is endowed with the analytic topology instead of the $C^{\infty}$ topology. Lemmas 1 and 3 need no change; The adjustment needed for the analytic topology in Lemma 2 was pointed out in the proof of that lemma. Parameter dependence still only needs to be $C^{2}$.

3. The theorem's result does not appear to hold if the family of homeomorphisms is assumed to be $C^{r}$ and is endowed with the $C^{r}$ topology because our proof of Lemma 3 requires the family to be $C^{r+1}$. If, however, we assume the family $F_{(\phi, \alpha)}(x)$ is $C^{r+1}$ as a function of $x$ but endow $\Upsilon$ with the $C^{r}$ topology, the theorem will hold. Parameter dependence still only needs to be $C^{2}$. 


\section{Comments}

\subsection{Implications for forced oscillators}

All the features we produced in this paper were using circle maps. There is a fairly standard construction in order to generate families of lifts of circle maps of the form of Eq. (1) from a periodically forced planar oscillator of form

$$
\frac{d \mathbf{x}}{d t}=\mathbf{V}(\mathbf{x})+\alpha \mathbf{W}(\mathbf{x}, \omega t)
$$

where $\mathbf{x} \in \mathbf{R}^{2} ; \omega, \alpha, \mathbf{t} \in \mathbf{R} ; \mathbf{W}$ is periodic with period one in its second variable; for $\alpha=0$ the nonautonomous flow has a repelling equilibrium point inside a normally hyperbolic attracting limit cycle. We have reviewed this construction in previous papers ([McGehee \& Peckham, 1994], [Peckham, 1990]).

The reverse process of generating a forced oscillator family from a family of circle map lifts of the form of Eq. (1) is also possible, but the process is not unique and not quite so straightforward. A sketch of one possible procedure is as follows. Put the original circle map family on the unit circle in $\mathbf{R}^{\mathbf{2}}$. Extend to a map of the plane by making the unit circle globally attracting, except for the origin, which is a repelling fixed point. Compose this family of maps of $\mathbf{R}^{\mathbf{2}}$ with a linear contraction with a globally contracting fixed point. Make the "contraction" the identity at $\alpha=0$ and of increasing contractivity as the forcing amplitude $\alpha$ is increased. (This and similar types of "impulse forcing" have been used by us as well as others in previous studies ([Schreiber, et. al., 1988], [Peckham, 1990]) to build caricatures of maps which are generated by periodically forced oscillators.) The composition results in a family of diffeomorphisms of $\mathbf{R}^{2}$ which can then be suspended to produce a flow in $\mathbf{R}^{\mathbf{2}} \times \mathbf{S}$. This flow can be thought of as the original periodically forced planar oscillator.

Because resonance regions for periodically forced oscillators typically close in a Hopf bifurcation at higher forcing amplitudes ([Peckham, 1990], [McGehee \& Peckham, 1994]), any extra orbits must eventually be eliminated as the forcing amplitude increases. Because extra orbits are generic at zero forcing amplitude, as established in the Theorem of Sec. 4, this establishes the genericity of flames in bifurcation diagrams for periodically forced oscillators. 


\subsection{Flames in applications}

We have presented in this paper a variety of bifurcation scenarios which might occur for periodically forced oscillators. On the other hand, the only feature which we have seen in numerical studies of periodically forced oscillators is the swallowtail. The question remains why flames, for example, are not widely seen in past numerical studies of periodically forced oscillators. There seem to be several possible answers. First, the features might actually be present in periodically forced oscillators that have been studied, even for low period resonances, but numerical work has just overlooked them. Second, the form of the periodically forced oscillators studied in the literature is such that the corresponding circle maps, like the standard family, are nongeneric and do not have features such as flames. Third, these features may exist in examples studied, but only in resonance regions with periods higher than those numerically studied.

Determining which of these answers applies in each case is not an easy task. It would require being able to calculate the coefficients of at least the lower mode coefficients in a Fourier series expansion for $g_{\alpha}$ in the circle map of Eq. (1) directly from the differential equation of the form of Eq. (14).

\subsection{Topology of resonance surfaces}

It turns out that the bifurcations described in this paper all deal with creating extra folds in the various resonance surfaces. While these folds are singularities with respect to the projection to the parameter plane, they are not topologically significant for the resonance surfaces. Work in progress considers resonance surfaces which have various numbers of topological handles. Such examples can be constructed via Eq. (1) by allowing $g_{(\phi, \alpha)}$ to actually depend on $\phi$.

\subsection{Acknowledgements}

The authors wish to thank G. R. Hall for enlightening and clarifying discussions on topics related to this paper. Thanks also to Alec Norton, who first suggested the possibility of the theorem we presented in Sec. 4.

This research was partially supported by NSF Grants DMS-9020220 and DMS-9206957. Both authors acknowledge the use of Geometry Center facil- 
ities and staff. 


\section{References}

Arnold, V.I. [1982] Geometrical Methods in the Theory of Ordinary Differential Equations, (Springer Verlag, New York).

Arnold, V.I. [1983] Russian Mathematical Surveys, 38215.

Aronson, D.G., McGehee, R.P., Kevrekidis, I.G. \& Aris, R. [1986], "Entrainment Regions for Periodically Forced Oscillators," Phys. Rev. A 33 (3), 2190-2192.

Aronson D.G., Chory M.A., Hall G.R., McGehee R.P. [1983], "Bifurcations from an invariant circle for two-parameter families of maps of the plane: a computer assisted study," Comm. Math. Phys., 83, 303-354.

Devaney R. L. [1989], An introduction to Chaotic Dynamical Systems, (AddisonWesley, New York).

Fagella, Nuria, "The Complex Standard Family," preprint.

Frouzakis C.E., Adomaitis R.A. and Kevrekidis I.G. [1991], "Resonance phenomena in an adaptivelly-controlled system", International Journal of Bifurcations and Chaos 1(1), 83-106.

Guckenheimer J and Holmes P [1983] Nonlinear Oscillations, Dynamical Systems and Bifurcations of Vector Fields, Applied Mathematical Sciences, 42 (Springer Verlag, New York).

Hall G.R. [1984], "Resonance Zones in two-parameter families of circle homeomorphisms," SIAM J. Math. Anal.15 (6), 1075-1081.

Kai T.\& Tomita K. [1979], "Stroboscopic phase portrait of a forced nonlinear oscillator," Progr. Theor. Phys., 61(1), 54-73.

Koslovsky O.S. [1991], "Resonance zone boundaries for families of circle diffeomorphisms," Physica D (54), 1-4. 
McGehee R.P. \& Peckham B.B. [1994], "Resonance Surfaces for Forced Oscillators," Geometry Center Research Report GCG70 and Experimental Mathematics 3(3), 221-244.

Peckham, B.B. [1990], "The Necessity of the Hopf Bifurcation for Periodically Forced oscillators with closed resonance regions," Nonlinearity (3), 261-280.

Peckham, B.B. [1988-94], To Be Continued ..., a continuation software package for discrete dynamical systems (continually under development).

Phillips et. al. [1993], Phillips M., Levy S. and Munzner T., "Geomview: An Interactive Geometry Viewer," Notices of the Amer. Math. Soc. 40 (1993), 985-988. This software and the accompanying manual are available by anonymous ftp from the host geom.umn.edu, in the directory $\mathrm{pub} /$ software.

Ruelle D. [1989], Elements of Differentiable Dynamics and Bifurcation Theory, (Academic Press, Inc., San Diego).

Schreiber I., Dolnik M., Choc P. \& Marek M. [1988], "Resonance Behaviour in Two-Parameter Families of Periodically Forced Oscillators," Physics Letters $A, \mathbf{1 2 8}, \mathbf{2}, 66-70$.

Yakobson M.V. [1985], "Number of periodic trajectories for analytic diffeomorphisms of the circle," Functional Analysis and its Applications, (19) 1, 79-80.

Wheeden R. L. \& Zygmund A. [1977], Measure and Integral, (Marcel Dekker, New York).

Zygmund, A., [1988, c1959] Trigonometric Series (Cambridge University Press, New York). 


\section{Complete Figure Captions}

1. A schematic of the $3 / 4$ resonance region from Fig. 3 of Schreiber, et. al. [1988]. The resonance region is in the $(T, A)$ parameter plane for a family of maps of the plane generated by a planar flow with "impulse forcing." The $T$ parameter can be thought of as controlling forcing frequency, the $A$ parameter as controlling forcing amplitude. Note especially the swallowtail in the $3 / 4$ resonance region.

2. (Reproduced from Fig. 6a of Frouzakis, et. al. [1991] with permission from the authors.) The $1 / 5$ resonance region in the $(k, b)$ parameter plane for a family of maps of $\mathbf{R}^{\mathbf{3}}$. Note especially the swallowtail in the resonance region.

3. Fixed points by graph. $F_{(-0.4,0.9)}(x)=x+(-0.4)+0.9\left(\left(0.7 \frac{\sin (2 x)}{2}+\right.\right.$ $0.3 \sin \left(2 \pi-h(.9) \frac{\pi}{2}\right)$, where $h(.9)=0.972$. The form of $F$ is chosen to match the form used later in the paper in Example 12.

4. Example 1, the Standard Family: $g(x)=\sin (x)$. The $0 / 1$ resonance surface and its projection to the parameter plane.

5. Example 1, the Standard Family: $g(x)=\sin (x)$. Several resonace surfaces and their projections to the parameter plane.

6. Example 2, Coincident projections: $g(x)=\sin (2 x)$.

7. Example 3, Noncoincident projections: $g(x)=0.9 \frac{\sin (2 x)}{2}+0.1 \sin (x)$.

8. Smooth bump functions $h(x)$ and $H(x)$.

9. Example 6, Single Flame: $g_{\alpha}(x)=(1-A(\alpha)) \frac{\sin (2 x)}{2}+A(\alpha) \sin (x)$, where $A(\alpha)=0.1+0.9 \alpha$. (a) The evolution of $g_{\alpha}(x): \alpha=0.1,0.5,0.9$. (b) The $0 / 1$ resonance surface and its projection to the parameter plane (with $\alpha$ replaced by $h\left(\alpha^{2}\right)$ ).

10. Example 7, Double Flame: $g_{\alpha}(x)=(1-h(\alpha)) \sin (3 x)+h(\alpha) \sin \left(x+\frac{\pi}{4}\right)$. The inset shows more clearly the double flame in the projection to the parameter plane. 
11. Example 9, Center Fold: $g_{\alpha}(x)=(1-A(\alpha)) \frac{\sin (2 x)}{2}+A(\alpha) \sin (x)$, where $A(\alpha)=1-0.7 H(\alpha)$.

12. Example 12, Left-side crossing: $g_{\alpha}(x)=0.7 \frac{\sin (2 x)}{2}+.3 \sin (x+2 \pi-$ $\left.\frac{\pi}{2} h(\alpha)\right)$. (a) The evolution of $g_{\alpha}(x): \alpha=0.1,0.5,0.9$. (b) The $0 / 1$ resonance surface and its projection to the parameter plane.

13. Example 13, Full twist: $g_{\alpha}(x)=0.7 \frac{\sin (2 x)}{2}+.3 \sin (x+2 \pi-2 \pi h(\alpha))$.

14. (a) The two-mode parameter space: $g_{\alpha}(x)=\frac{1-A}{2} \sin (2 x)+A \sin (x)$. (b) The path through this two-mode parameter space which corresponds to the left edge swallowtail of Example 15.

15. Example 15, the left edge swallowtail: $g_{\alpha}(x)=\frac{(1-A(\alpha))}{2} \sin (2 x)+A(\alpha) \sin (x+$ $B(\alpha))$, with $A(\alpha)=1-.7 H(\alpha)$, and $B(\alpha)=2 \pi-\frac{\pi}{2} h(\alpha)$. 